\title{
Uso de cristaloides en la reanimación del paciente crítico
}

\author{
Cristalloids in critically patients
}

Felipe Muñoz ${ }^{1}$ Lucia Arratia², Eduardo Kattan²

\begin{abstract}
Introduction: Fluid therapy is a very frequent intervention in critically ill patients. Fluids can be divided into crystalloids and colloids, being the former among the most widely used. Within crystalloids, we can further divide them into balanced and unbalanced. There is some evidence pointing towards a benefit from using balanced crystalloids over unbalanced crystalloids in acid-base balance, kidney failure, and mortality, but it is not clear whether this is clinically relevant. Methods: We performed a search in the PUBMED and EMBASE databases and filtered for studies performed in the last ten years which included critically ill patients. Results: We reviewed nine randomized clinical trials and four retrospective studies. Balanced fluids probably have a beneficial effect in preventing hyperchloremic metabolic acidosis, and they could also reduce kidney failure and the composite outcome MAKE30. Mortality reduction was not statistically significant. The available evidence to evaluate the effect on hemodynamic stability, systemic inflammatory response syndrome (SIRS) reduction, C-reactive protein reduction, days free of vasopressors, mechanical ventilation, and others, is insufficient. Conclusion: Balanced crystalloids are a valid alternative to unbalanced crystalloids with several potential benefits, therefore they should be preferred if possible. More evidence is needed to determine the possible benefit on mortality.
\end{abstract}

\section{RESUMEN}

Introducción: La reanimación con fluidos es una intervención muy frecuente en pacientes críticos. Los fluidos pueden dividirse en cristaloides y coloides, siendo los primeros los más usados en la práctica clínica. En este grupo, se
Key words:

Crystalloids, balanced fluids, normal saline

Palabras clave:

Cristaloides, balance fluidos, solución fisiológica

Interno de Medicina, Escuela de Medicina, Facultad de Medicina, Pontificia Universidad Católica de Chile.

Departamento de Medicina Intensiva, Facultad de Medicina, Pontificia Universidad Católica de Chile.

Fecha de ingreso: 03 de agosto de 2020

Fecha de aceptación: 12 de octubre de 2020

\section{ORCID}

0000-0002-1997-6893

Conflictos de interés: Los autores no presentan conflicto de interés.

Correspondencia:

Eduardo Kattan MD; MMEd

Departamento de Medicina Intensiva, Facultad de Medicina, Pontificia Universidad Católica de Chile.

e.kattan@gmail.com 
pueden encontrar cristaloides balanceados y no balanceados. Existe cierta evidencia de que los cristaloides balanceados podrían tener beneficios sobre los no balanceados en cuanto a estado ácido base, falla renal y mortalidad, sin embargo, no está claro que estos beneficios sean significativos en la práctica clínica. Métodos: Se realizó una búsqueda en las bases de datos de PUBMED y EMBASE utilizando como términos de búsqueda "crystalloid", "fluid therapy", "balanced fluid", "saline", "intravenous fluid", "ringer's lactate" y "plasmalyte" y se filtró por los estudios realizados en los últimos diez años que incluyeran pacientes críticos. Resultados: Se revisaron nueve ensayos clínicos aleatorizados y cuatro estudios retrospectivos. Los fluidos balanceados probablemente tienen un efecto beneficioso en evitar la acidosis metabólica hiperclorémica y podrían reducir la falla renal y el desenlace compuesto de eventos adversos renales mayores (MAKE30). La reducción de mortalidad no fue significativa estadísticamente. La evidencia disponible es insuficiente para la evaluación de otros desenlaces como estabilidad hemodinámica, reducción del síndrome inflamatorio de respuesta sistémica (SIRS), proteína C reactiva, días libres de vasopresores, ventilación mecánica, entre otros. Conclusiones: Las soluciones balanceadas se presentan como una alternativa con múltiples beneficios probables sobre las soluciones no balanceadas. Se requiere mayor evidencia para evaluar qué tipo de pacientes se beneficiarían más de su utilización y determinar el posible beneficio en desenlaces como mortalidad.

\section{Introducción}

a administración de fluidos endovenosos es una de las intervenciones más frecuentemente realizadas en las unidades de cuidados intensivos a lo largo del mundo[1],[2]. El fluido ideal para la reanimación debería producir una mejoría predecible del volumen estresado, con una composición química lo más similar al plasma, ser metabolizado y excretado sin acumularse en los tejidos, no producir efectos adversos metabólicos ni sistémicos, a un precio razonable, y ser costo efectivo en mejorar los resultados clínicos de los pacientes[1]. Lamentablemente, en la actualidad tal fluido no existe, por lo que, se deben conocer las características, ventajas y desventajas de los distintos tipos de fluidos disponibles en el ámbito intrahospitalario.

En términos generales, los fluidos utilizados en la práctica clínica pueden ser divididos en coloides, los cuales corresponden a soluciones que presentan proteínas transportadoras en suspensión incapaces de traspasar la membrana capilar, y cristaloides, que corresponden a soluciones de iones que pueden traspasar libremente la membrana capilar, pero que a través de sus iones fuertes mantienen cierta tonicidad respecto al plasma[1],[2]. En la atención hospitalaria habitual, tanto por costos como por disponibilidad y evidencia actual, los cristaloides son los fluidos más utilizados en la resucitación de pacientes a nivel mundial[2].

Los cristaloides pueden dividirse de forma general en soluciones no balanceadas y balanceadas, dependiendo de la similitud de la solución con la composición electrolítica del plasma[2]. Las soluciones balanceadas, tales como Ringer lactato y Plasma-Lyte ${ }^{\circledR}$ (Baxter International Inc; Illinois, EEUU), utilizan precursores de bicarbonato para simular las condiciones del plasma, al ser estos metabolizados a bicarbonato una vez que se infunde el fluido y de esta forma alcalinizar el plasma[2]. Por otro lado, se encuentran las soluciones no balanceadas, representadas en la práctica clínica habitual por la solución salina 0,9\%, también (mal) llamada suero fisiológico. Esta última posee una concentración de ion cloro más elevada que la del plasma (154 mEq/L vs 100 mEq/L) y, además, una diferencia de iones fuertes de cero, en comparación al plasma, que presenta una diferencia de aproximadamente $40 \mathrm{mEq} / \mathrm{L}[2]$. En la Tabla 1 se presenta la composición químicas de los principales cristaloides disponibles en el mercado.

A raíz de las diferencias en la composición entre ambos tipos de fluidos surge la interrogante sobre el posible beneficio clínico en el uso de un tipo de 


\begin{tabular}{lcccc}
\multicolumn{5}{c}{ Tabla 1. Composición de los cristaloides revisados } \\
\hline & Solución salina 0,9\% & $\begin{array}{c}\text { Solución Ringer } \\
\text { Lactato }\end{array}$ & Plasma-Lyte A/148 ${ }^{\circledR}$ & Normosol $^{\circledR}$ \\
Osmolaridad (mEq/L) & 308 & $273-277$ & 295 & 295 \\
$\mathrm{pH}($ aprox $)$ & $5,0-7,0$ & $6,0-7,3$ & 7,4 & 7,4 \\
$\mathrm{Na}(\mathrm{mEq} / \mathrm{L})$ & 154 & 130 & 140 & 140 \\
$\mathrm{Cl}(\mathrm{mEq} / \mathrm{L})$ & 154 & $109-111$ & 98 & 98 \\
$\mathrm{~K}(\mathrm{mEq} / \mathrm{L})$ & 0 & $4,0-5,4$ & 5,0 & 5,0 \\
$\mathrm{Mg}(\mathrm{mEq} / \mathrm{L})$ & 0 & 0 & 3,0 & 3,0 \\
$\mathrm{Ca}(\mathrm{mEq} / \mathrm{L})$ & 0 & 3,7 & 0 & 0 \\
Acetato $(\mathrm{mEq} / \mathrm{L})$ & 0 & 0 & 27 & 27 \\
Lactato $(\mathrm{mEq} / \mathrm{L})$ & 0 & 27,8 & 0 & 0 \\
Gluconato $(\mathrm{mEq} / \mathrm{L})$ & 0 & 0 & 23 & 23 \\
\hline
\end{tabular}

fluidos sobre otro. Múltiples estudios no randomizados y retrospectivos han concluido que las soluciones salinas tradicionales se asocian con mayores efectos adversos como acidosis metabólica hiperclorémica[3], falla renal[4],[5] e incluso mortalidad intrahospitalaria[6]. A diferencia de estas, las soluciones balanceadas podrían evitar esos efectos al presentar una composición más similar a la del plasma y mantener un equilibrio hidroelectrolítico adecuado.

El objetivo de esta revisión es comparar el uso de soluciones balanceadas con las soluciones salinas en la reanimación de pacientes críticos, según el estado actual de la evidencia, con énfasis en la búsqueda de estudios clínicos randomizados que puedan otorgar un mayor nivel de evidencia que permita mayor aplicabilidad a la práctica clínica.

Para lograr este objetivo, se realizó una búsqueda en la base de datos PUBMED y EMBASE utilizando como términos de búsqueda "crystalloid", "fluid therapy", "balanced fluid", "saline", "intravenous fluid", "ringer's lactate" y "plasmalyte" y se filtró por aquellos estudios que se realizaron en los últimos diez años, que correspondían a estudios primarios y que incorporaban reanimación con fluidos en pacientes críticos, de esta forma se obtuvieron cinco estudios retrospectivos[6],[7],[8],[9],[10] y nueve ensayos clínicos randomizados[11],[12],[13],[14],[15],[16],[17],[18],[19] (Tabla 2) cuyos resultados se analizarán de forma cualitativa en cuanto al impacto en el estado ácido base, falla renal, mortalidad y otros, se propondrán posibles asociaciones en base a la evidencia disponible y finalmente se plantearán conclusiones sobre la selección de terapia de fluidos en pacientes críticos en base a este análisis.

\section{Estado ácido base}

La aparición de acidosis metabólica hiperclorémica posterior a la resucitación con fluidos ha sido motivo de estudio por décadas. El modelo de regulación ácido base más aceptado hoy en día corresponde al propuesto por Stewart en 1980, que sugiere que el pH plasmático está determinado por 3 factores principales; la diferencia de iones fuertes (cationes $\mathrm{Na}^{+}, \mathrm{K}^{+}$, $\mathrm{Ca}^{2+}$ y $\mathrm{Mg}^{+}$y aniones $\mathrm{Cl}^{-}$, lactato y cetonas), la presión parcial de $\mathrm{CO}_{2}$ y la concentración de ácidos débiles no volátiles, como albúmina y fosfato[20].

La presencia de hipercloremia produce una disminución de la diferencia de iones fuertes, generando una caída del pH plasmático y por tanto acidosis. Esta acidosis hiperclorémica, produce un efecto proinflamatorio, se asocia a mayor inestabilidad hemodinámica y también a mayor falla renal[7],[20]. A partir de estos hallazgos, se puede inferir que la infusión de fluidos endovenosos, como solución salina, que presentan una diferencia de iones fuertes de cero, pueden producir mayor hipercloremia y por tanto mayor acidosis metabólica, a diferencia de las soluciones balanceadas.

En base a esto, tanto en estudios retrospectivos no randomizados como en estudios clínicos randomizados se han encontrado diferencias estadísticamente significativas en cuanto a los niveles de cloro alcanzados en aquellos pacientes, siendo mayores en quienes recibieron solución salina que en los pacientes asignados al grupo de soluciones balanceadas[8],[9],[10],[11],,[12],[13],[14]. Además, la incidencia de acidosis metabólica también fue mayor en pacientes tratados con solución salina comparados 
Tabla 2. Ensayos clínicos randomizados incluidos en la revisión

\begin{tabular}{|c|c|c|c|c|c|c|}
\hline Referencia & $\begin{array}{l}\text { Nombre del } \\
\text { estudio }\end{array}$ & $\begin{array}{l}\text { Tipo de es- } \\
\text { tudio }\end{array}$ & $\mathrm{n}$ & Intervención & Comparación & $\begin{array}{l}\text { Resultados } \\
\text { principales }\end{array}$ \\
\hline $\begin{array}{l}\text { Young J, et al } \\
\text { (2014) [11] }\end{array}$ & $\begin{array}{l}\text { Saline versus Plas- } \\
\text { ma-Lyte } A \text { in Initial } \\
\text { Resuscitation of } \\
\text { Trauma Patients }\end{array}$ & $\begin{array}{l}\text { Ensayo clínico } \\
\text { randomizado } \\
\text { unicéntrico }\end{array}$ & 46 & $\begin{array}{l}\text { Plasma-Lyte A } \\
\text { (PL-A) }\end{array}$ & $\begin{array}{l}\text { Solución sali- } \\
\text { na } 0,9 \%(\mathrm{NaCl} \\
0,9 \%)\end{array}$ & $\begin{array}{l}\text { Mejoría promedio en exceso de } \\
\text { base en } 24 \mathrm{~h} \text { significativamente } \\
\text { superior con PL-A }(p<0,001) \\
\text { pH arterial en } 24 \mathrm{~h} \text { significati- } \\
\text { vamente superior con PL-A ( } p= \\
0,003) \\
\text { Cloro plasmático en } 24 \mathrm{~h} \text { signi- } \\
\text { ficativamente superior con } \mathrm{NaCl} \\
0,9 \% \text { ( } p<0,001 \text { ) } \\
\text { Magnesio plasmático en } 24 \mathrm{~h} \\
\text { significativamente superior con } \\
\text { PL-A ( } p=0,007) \\
\text { Otros electrolitos plasmáticos, } \\
\text { balance de fluidos, utilización de } \\
\text { recursos y mortalidad intrahos- } \\
\text { pitalaria no fueron significativos }\end{array}$ \\
\hline $\begin{array}{l}\text { Verma B, et al } \\
\text { (2016) [12] }\end{array}$ & $\begin{array}{l}\text { A multicentre, ran- } \\
\text { domised controlled } \\
\text { pilot study of fluid } \\
\text { resuscitation with } \\
\text { saline or Plasma- } \\
\text { Lyte } 148 \text { in critica- } \\
\text { lly ill patients }\end{array}$ & $\begin{array}{l}\text { Ensayo clínico } \\
\text { randomizado } \\
\text { multicéntrico }\end{array}$ & 70 & $\begin{array}{l}\text { Plasma-Lyte } 148 \\
\text { (PL-148) }\end{array}$ & $\begin{array}{l}\text { Solución sali- } \\
\text { na } 0,9 \% \quad(\mathrm{NaCl} \\
0,9 \%)\end{array}$ & $\begin{array}{l}\text { Cloro plasmático significativa- } \\
\text { mente superior con } \mathrm{NaCl} 0,9 \% \\
(p=0,01) \\
\text { Exceso de base en los primeros } \\
\text { cuatro días, incidencia de falla } \\
\text { renal, variación de creatinina y } \\
\text { mortalidad no fueron significa- } \\
\text { tivos }\end{array}$ \\
\hline $\begin{array}{l}\text { Semler M, et } \\
\text { al (2018) [13] }\end{array}$ & $\begin{array}{l}\text { Balanced Crysta- } \\
\text { lloids versus Sali- } \\
\text { ne in Critically III } \\
\text { Adults (SMART } \\
\text { trial) }\end{array}$ & $\begin{array}{l}\text { Ensayo clínico } \\
\text { rando mizado } \\
\text { unicéntrico }\end{array}$ & 15.802 & $\begin{array}{l}\text { Ringer lactato o } \\
\text { Plasma-Lyte A } \\
\text { (cristaloides ba- } \\
\text { lanceados) }\end{array}$ & $\begin{array}{l}\text { Solución sali- } \\
\text { na } 0,9 \% \quad(\mathrm{NaCl} \\
0,9 \%)\end{array}$ & $\begin{array}{l}\text { MAKE30 significativamente su- } \\
\text { perior con } \mathrm{NaCl} 0,9 \%(p=0,04) \\
\text { Días libres de TRR significativa- } \\
\text { mente superior con cristaloides } \\
\text { balanceados }(p=0,01) \\
\text { Mortalidad intrahospitalaria, a } \\
30 \text { días, a } 60 \text { días, días libres de } \\
\text { UCl, VMl y vasopresores no fue- } \\
\text { ron significativos }\end{array}$ \\
\hline $\begin{array}{l}\text { Semler M, et } \\
\text { al (2017) [14] }\end{array}$ & $\begin{array}{l}\text { Balanced Crysta- } \\
\text { lloids versus Saline } \\
\text { in the Intensive } \\
\text { Care Unit. The } \\
\text { SALT Randomized } \\
\text { Trial }\end{array}$ & $\begin{array}{l}\text { Ensayo clínico } \\
\text { randomizado } \\
\text { unicéntrico }\end{array}$ & 974 & $\begin{array}{l}\text { Ringer lactato o } \\
\text { Plasma-Lyte A } \\
\text { (cristaloides ba- } \\
\text { lanceados) }\end{array}$ & $\begin{array}{l}\text { Solución sali- } \\
\text { na } 0,9 \% \quad(\mathrm{NaCl} \\
0,9 \%)\end{array}$ & $\begin{array}{l}\text { Proporción de cristaloides co- } \\
\text { rrespondientes a solución salina } \\
\text { entre ambos grupos significati- } \\
\text { vamente superior en grupo } \mathrm{NaCl} \\
0,9 \%(\mathrm{p}<0,001) \\
\text { MAKE30 no fue significativo } \\
\text { En análisis de subgrupo MAKE30 } \\
\text { en mayor volumen de cristaloi- } \\
\text { des significativamente mayor } \\
\text { con } \mathrm{NaCl} 0,9 \text { (p para interacción } \\
=0,026)\end{array}$ \\
\hline $\begin{array}{l}\text { Young } P \text {, et al } \\
\text { (2015) [15] }\end{array}$ & $\begin{array}{l}\text { Effect of a Buffe- } \\
\text { red Crystalloid So- } \\
\text { lution vs Saline on } \\
\text { Acute Kidney Injury } \\
\text { Among Patients in } \\
\text { the Intensive Care } \\
\text { Unit: The SPLIT } \\
\text { Randomized Clini- } \\
\text { cal Trial }\end{array}$ & $\begin{array}{l}\text { Ensayo clínico } \\
\text { rando mizado } \\
\text { multicéntrico }\end{array}$ & 2.278 & $\begin{array}{l}\text { Plasma-Lyte } 148 \\
\text { (PL-148) }\end{array}$ & $\begin{array}{l}\text { Solución sali- } \\
\text { na } 0,9 \%(\mathrm{NaCl} \\
0,9 \%)\end{array}$ & $\begin{array}{l}\text { Falla renal, incidencia de TRR y } \\
\text { mortalidad intrahospitalaria no } \\
\text { fueron significativos }\end{array}$ \\
\hline
\end{tabular}




\begin{tabular}{|c|c|c|c|c|c|c|}
\hline $\begin{array}{l}\text { Choosakul S, } \\
\text { et al (2018) } \\
{[16]}\end{array}$ & $\begin{array}{l}\text { Comparison of } \\
\text { normal saline } \\
\text { versus Lactated } \\
\text { Ringer's solution } \\
\text { for fluid resusci- } \\
\text { tation in patients } \\
\text { with mild acute } \\
\text { pancreatitis, A ran- } \\
\text { domized controlled } \\
\text { trial }\end{array}$ & $\begin{array}{l}\text { Ensayo clínico } \\
\text { rand o mizado } \\
\text { unicéntrico }\end{array}$ & 47 & $\begin{array}{l}\text { Ringer lactato } \\
\text { (RL) }\end{array}$ & $\begin{array}{l}\text { Solución sali- } \\
\text { na } 0,9 \% \quad(\mathrm{NaCl} \\
0,9 \%)\end{array}$ & $\begin{array}{l}\text { Reducción SIRS a } 24 \mathrm{~h} \text { significa- } \\
\text { tivamente superior con RL ( } p= \\
0,02 \text { ) } \\
\text { Reducción SIRS a } 48 \mathrm{~h} \text {, morta- } \\
\text { lidad, complicaciones locales } \\
\text { y parámetros inflamatorios no } \\
\text { fueron significativos }\end{array}$ \\
\hline $\begin{array}{l}\text { Wang X, et al } \\
\text { (2016) [17] }\end{array}$ & $\begin{array}{l}\text { Association bet- } \\
\text { ween chloride- } \\
\text { rich versus chlo- } \\
\text { ride-restrictive } \\
\text { intravenous fluid } \\
\text { administration and } \\
\text { acute kidney injury } \\
\text { in cardiovascular } \\
\text { patients in ICU } \\
\text { wards }\end{array}$ & $\begin{array}{l}\text { Ensayo clínico } \\
\text { rando mizado } \\
\text { unicéntrico }\end{array}$ & 456 & $\begin{array}{l}\text { Ringer lactato o } \\
\text { albúmina } 20 \% \\
\text { (grupo bajo en } \\
\text { cloro) }\end{array}$ & $\begin{array}{l}\text { Solución salina } \\
0,9 \% \text { o albúmi- } \\
\text { na } 4 \% \text { en clo- } \\
\text { ruro de sodio o } \\
\text { gelatinas (grupo } \\
\text { alto en cloro) }\end{array}$ & $\begin{array}{l}\text { Incidencia de falla renal KDIGO } 2 \\
\text { y } 3 \text { significativamente menor en } \\
\text { grupo bajo en cloro }(p<0,05) \\
\text { Incidencia de falla renal KDIGO } \\
3 \text { significativamente menor en } \\
\text { grupo bajo en cloro }(p<0,05)\end{array}$ \\
\hline $\begin{array}{l}\text { de-Madaria E, } \\
\text { et al (2018) } \\
{[18]}\end{array}$ & $\begin{array}{l}\text { Fluid resuscita- } \\
\text { tion with lactated } \\
\text { Ringer's solution } \\
\text { vs normal saline in } \\
\text { acute pancreatitis: } \\
\text { A triple-blind, ran- } \\
\text { domized, contro- } \\
\text { lled trial }\end{array}$ & $\begin{array}{l}\text { Ensayo clínico } \\
\text { rando mizado } \\
\text { unicéntrico }\end{array}$ & 40 & Ringer lactato & $\begin{array}{l}\text { Solución salina } \\
0,9 \%\end{array}$ & $\begin{array}{l}\text { Reducción de SIRS a } 48 \text { h y } 72 \\
\text { h con tendencia no estadística- } \\
\text { mente significativa con RL ( } p= \\
0,06 \text { y } p=0,064 \text { ) } \\
\text { Reducción de PCR a } 48 \text { h y } 72 \\
\text { h significamente superior con RL } \\
\text { ( } p=0,037 \text { y } p=0,043 \text { ) } \\
\text { Bicarbonato plasmático signifi- } \\
\text { cativamente superior con RL } \\
\text { Incidencia de complicaciones } \\
\text { locales y sistémicas, de enfer- } \\
\text { medad moderada a severa y pH } \\
\text { sanguíneo no estadísticamente } \\
\text { significativos }\end{array}$ \\
\hline $\begin{array}{l}\text { Wu B, et al } \\
(2011)[19]\end{array}$ & $\begin{array}{l}\text { Lactated Ringer's } \\
\text { solution reduces } \\
\text { systemic inflam- } \\
\text { mation compared } \\
\text { with saline in pa- } \\
\text { tients with acute } \\
\text { pancreatitis }\end{array}$ & $\begin{array}{l}\text { Ensayo clínico } \\
\text { rand o mizado } \\
\text { multicéntrico }\end{array}$ & 40 & Ringer lactato & $\begin{array}{l}\text { Solución salina } \\
0,9 \%\end{array}$ & $\begin{array}{l}\text { Reducción SIRS y PCR a } 24 \mathrm{~h} \text { sig- } \\
\text { nificativamente superior con RL } \\
(p=0,035 \text { y } p=0,018) \\
\text { Incidencia de acidosis metabóli- } \\
\text { ca significativamente menor con } \\
\text { RL ( } p=0,01 \text { ) } \\
\text { Duración de estadía hospitalaria, } \\
\text { complicaciones locales y sistémi- } \\
\text { cas no estadísticamente signifi- } \\
\text { cativos }\end{array}$ \\
\hline
\end{tabular}

MAKE30: Outcome compuesto de mortalidad, diálisis y falla renal persistente.

con aquellos que recibieron soluciones balanceadas, tanto en algunos de estos estudios retrospectivos como en algunos estudios clínicos randomiza$\operatorname{dos}[9],[10],[11],[13],[19]$.

Es importante tener en consideración que estos desenlaces reportados de variaciones en la cloremia como en la incidencia de acidosis metabólica, corresponden en algunos estudios sólo a pacientes sépticos o cursando un cuadro de cetoacidosis diabética, que por la presencia de hiperlactatemia o cetonas podrían ver alterada la interpretación de estos resultados[8],[9],[10]. Se debe considerar también que algunos de los estudios que no señalan una variación de los niveles de cloro o la presencia de acidosis, simplemente no consideraron estos un resultado a evaluar[15],[16],[17]. De todas formas, la gran mayoría de los estudios aprecia una disminución significativa tanto de la hipercloremia como de la acidosis con el 
uso de soluciones balanceadas, lo que podría incidir en una mejoría del estado ácido base y prevención de complicaciones como falla renal[7],[20].

\section{Falla renal}

La falla renal aguda es muy frecuente en el contexto del paciente crítico, llegando a presentar una incidencia de más del $50 \%$ en las unidades de paciente crítico (UPC)[21]. Ésta se define como un deterioro de la función renal marcado por un rápido aumento de la creatinina plasmática, caída de la diuresis o ambos[21]. En los pacientes críticos la etiología de esta falla renal es multifactorial, incluyendo estados de hipovolemia y shock con hipoperfusión, sepsis, uso de drogas nefrotóxicas, entre otras[21].

Es en este contexto, el de un paciente crítico cursando cuadros sépticos o hemorrágicos que pueden asociarse a hipovolemia o shock, donde la resucitación inicial con fluidos puede contribuir a la disminución de la incidencia o progresión de falla renal. ${ }^{21}$ Bajo esta premisa y dado que el uso de fluidos no balanceados genera alteraciones ácido base que pudieran favorecer la aparición de falla renal, es donde el uso de fluidos balanceados podría tener un rol en la prevención de ésta.

Dentro de los estudios evaluados, un estudio retrospectivo y un estudio clínico randomizado encontraron una disminución significativa de la incidencia de falla renal con el uso de soluciones restrictivas en cloro, las cuales corresponden en su totalidad a soluciones balanceadas[10],[17]. Cabe destacar que en el estudio randomizado se utilizaron coloides además de cristaloides, corresponde solo a pacientes cardiovasculares y el análisis estadístico no está completamente disponible. Otro estudio clínico randomizado, el SMART Clinical Trial, obtuvo en sus resultados una diferencia significativa en el desenlace compuesto de eventos adversos renales mayores a 30 días (MAKE30), a favor del uso de soluciones balanceadas[13]. Esta diferencia fue aún más significativa en pacientes reanimados con mayores volúmenes de cristaloides y en pacientes sépticos[13].

Por otro lado, tres estudios retrospectivos y cuatro estudios clínicos randomizados reportaron una variación no significativa en la incidencia de falla renal aguda al variar el tipo de cristaloide utilizado[6],[8],[9],[11],[12],[14],[15]. Otro estudio clínico randomizado revisado no evaluó la incidencia de falla renal dentro de sus resultados[16]. Es importante tener en consideración que el número de participantes de tres de estos estudios clínicos randomizados no superó los 70 participantes en cada uno[11],[12],[16].

Dos de los estudios clínicos randomizados que resultaron no significativos para este desenlace, a pesar de ser ciegos para los médicos tratantes en cuanto al fluido utilizado, si permitieron al clínico disponer de la información de laboratorio respecto a gasometría y medición de electrolitos plasmáticos, lo que podría haber limitado la hipercloremia, acidosis metabólica y progresión a falla renal[11],[12]. Otro de los estudios clínicos randomizados no significativos, el estudio SPLIT, presentó una exposición de más del 90\% de sus participantes a fluidos balanceados previo a la incorporación al estudio, lo cual pudo haber limitado la significancia de todos sus resultados evaluados. ${ }^{15}$ De todas formas, la evidencia actual, considerando los estudios evaluados, se inclina a considerar un potencial beneficio en el uso de soluciones balanceadas para la prevención de la aparición o progresión de falla renal.

\section{Efectos sobre la mortalidad}

Uno de los desenlaces más relevantes dentro de los estudios que incluyen pacientes críticos, ha sido la reducción de la mortalidad como desenlace centrado en el paciente. Se debe tener en consideración que los pacientes críticos, dada la gravedad de su patología podrían ver influenciados los desenlaces de mortalidad por intervenciones que en otros pacientes podrían no ser significativas.

Por otro lado, la disminución de la mortalidad debido a otras intervenciones, la multifactorialidad de la muerte, los esfuerzos del equipo de salud de las UPC, la necesidad de tamaños muestrales progresivamente más grandes, hacen cada vez más difícil demostrar una disminución de la mortalidad significativa[22],[23],[24]. Es por esto, que diversas publicaciones han sugerido la necesidad de reconsiderar la evaluación de mortalidad como resultado principal en este tipo de estudios[22],[23],[24]. En esta misma línea, en cuanto a la terapia de fluidos, hay estudios que demuestran que el volumen de fluidos utilizado durante la fase de reanimación corresponden a un porcentaje menor del total de fluidos recibidos durante la estadía en UCI[25], lo que podría causar mayor dificultad en la evaluación del impacto sobre la mortalidad en los pacientes críticos.

Solo un estudio retrospectivo ha mostrado variaciones significativas en cuanto a mortalidad intrahospitalaria, a favor del uso de soluciones balanceadas por sobre no balanceadas[6]. Se debe destacar que en este estudio participaron 360 unidades de paciente 
crítico, pero dado el diseño del estudio y la recolección electrónica de datos de fichas clínicas, no se dispone de mediciones de laboratorio ni otros factores que pudieran haber influenciado a los médicos tratantes en la elección de cristaloides.

Ningún estudio clínico randomizado que ha reportado mortalidad dentro de sus resultados ni dos de los estudios retrospectivos evaluados han encontrado diferencias estadísticamente significativas en el uso de cristaloides balanceados o no balanceados en la reanimación de pacientes críti$\cos [8],[10],[11],[12],[13],[14],[15],[16]$.

A pesar de esto, se debe tener en consideración que en el estudio SMART, el estudio clínico randomizado con mayor número de participantes evaluados, diseño metodológico robusto, y uno de los más recientes, la mortalidad intrahospitalaria a 30 días bordeó la significancia estadística a favor del uso de soluciones balanceadas $(10,3 \%$ vs $11,1 \%, p=0,06)$ [13]. Esto podría insinuar un eventual beneficio, o al menos no inferioridad, en el uso de soluciones balanceadas respecto a la mortalidad intrahospitalaria. El estudio PLUS o el estudio BaSICS, ambos actualmente en curso, que tienen por desenlace primario evaluar mortalidad, podrían otorgar mayor evidencia respecto al eventual beneficio de las soluciones balanceadas[26],[27].

\section{Otros resultados respecto al uso de fluidos}

Dentro de los estudios evaluados en esta revisión también se han reportado otros desenlaces que pueden ser de utilidad al momento de decidir respecto al uso de cristaloides balanceados o no balanceados para la reanimación de pacientes críticos.

Un estudio retrospectivo reportó mayor estabilidad de los parámetros hemodinámicos con el uso de soluciones balanceadas en pacientes cursando cetoacidosis, lo cual podría ser concordante con los efectos observados de la hipercloremia en otros estudios[7],[10]. Ningún otro estudio consideró la evaluación hemodinámica dentro de sus resultados reportados, por lo que se requieren más estudios para poder considerarlo un efecto significativo en la práctica clínica habitual.

Dos estudios clínicos randomizados consignaron una disminución estadísticamente significativa del síndrome inflamatorio de respuesta sistémica (SIRS) a las 24 h, a favor del uso de cristaloides balanceados en pacientes cursando pancreatitis[16],[19], mientras que un tercer estudio clínico randomizado reportó una variación no estadísticamente significativa[18].
Asimismo, dos estudios clínicos randomizados encontraron una disminución estadísticamente significativa de la proteína $C$ reactiva ( $P C R$ ) en pacientes con pancreatitis a favor de cristaloides balanceados, en un estudio a 24 h y en otro a 48 y 72 h[18],[19]. Dado el tamaño muestral y las limitaciones de los estudios se requiere mayor evidencia para poder evaluar la utilidad práctica de este posible beneficio.

Otros resultados evaluados en estos estudios fueron duración de estadía hospitalaria o en $\mathrm{UCl}$, lo que no resultó en variaciones significativas en ningún estudio[6],[9],[10],[11],[12],[13],[14],[15],[16]. La evaluación de días libres de vasopresores, ventilación mecánica o terapia de reemplazo renal tampoco resultó significativa en ninguno de los estudios que reportó estos resultados[12],[13],[14],[15]. Todos éstos son medidas indirectas del impacto de una intervención y dependen de múltiples otros factores, lo que pudiera explicar que ninguno de ellos resultara estadísticamente significativo.

\section{Discusión y Conclusiones}

En base a la bibliografía revisada, se puede concluir que los cristaloides balanceados podrían tener un efecto beneficioso en la mejoría del estado ácido base del paciente al evitar la acidosis metabólica hiperclorémica. También podrían ser beneficios en disminuir la incidencia de falla renal, evaluada en desenlaces compuestos como el MAKE30, lo cual podría tener aún mayor impacto en pacientes que reciben grandes volúmenes de cristaloides y en paciente sépticos.

Respecto a los efectos sobre mortalidad, el uso de cristaloides balanceados es al menos no inferior al uso de cristaloides no balanceados, pero con tendencia hacia un posible efecto beneficioso. Por otro lado, en cuanto a otros resultados evaluados por los estudios de esta revisión, tales como estabilidad hemodinámica, disminución del SIRS o PCR, la evidencia no es concluyente, aunque pareciese existir un posible beneficio en la reducción de inflamación en patologías como pancreatitis, pero se requiere mayor evidencia para poder evaluar su impacto clínico.

Se necesitan más estudios clínicos para poder determinar finalmente si existen beneficios en la mortalidad al utilizar cristaloides balanceados, como los estudios PLUS o BaSICS, que se encuentran en curso, para determinar posibles efectos adversos de éstos que no hayan sido evaluados previamente y también para evaluar la variabilidad que pueda tener el uso de distintas soluciones balanceadas en los desenlaces descritos previamente. 


\section{Mitos respecto al uso de fluidos balanceados}

Otro aspecto importante a considerar en la selección de fluidoterapia, es la necesidad de la administración concomitante con transfusiones de hemoderivados. Tradicionalmente, se considera que soluciones que poseen $\mathrm{Ca}^{2+}$, como Ringer lactato, podrían inducir la coagulación de estos hemoderivados y por tanto debiera utilizarse una vía venosa separada. A pesar de la base teórica detrás, existe diversa evidencia de que sería seguro utilizar Ringer lactato en conjunto con transfusión de hemoderivados y además, se debe considerar que otros fluidos balanceados como PlasmaLyte $^{\circledR}$ y Normosol ${ }^{\circledR}$ no contienen calcio[28],[29],[30].

En cuanto al uso en pacientes que presentan falla renal, existe la creencia de que el uso de soluciones balanceadas, que contienen potasio entre 4,0 y 5,4 $\mathrm{mEq} / \mathrm{L}$, podrían causar hiperkalemia. Al contrario de esta creencia, la evidencia indica que la resucitación con solución salina induce mayor hiperkalemia comparado con Ringer lactato, causado por su diferencia de iones fuertes de cero, lo que actúa como un mecanismo de shift de potasio hacia el plasma[31],[32]. A modo de ejemplo, para que la administración de soluciones balanceadas tuviesen un efecto similar en cuanto al aporte de potasio al de una ampolla de cloruro de potasio (que contiene 13,4 $\mathrm{mEq}$ ), el paciente debe recibir al menos tres litros de fluidos. Por ende, incluso en pacientes con insuficiencia renal, las soluciones balanceadas parecen ser seguras.

\section{Limitantes de la revisión}

Esta revisión posee varias limitaciones. La búsqueda de información no se realizó a través de un protocolo sistematizado y solo se incluyeron dos bases de datos relacionadas con el área de Salud, por lo que es posible que exista más evidencia disponible no incorporada en esta revisión. El análisis de esta revisión fue realizado de forma narrativa, sin incorporar un meta-análisis que otorgue conclusiones objetivas respecto a los datos analizados. Los tamaños muestrales necesarios para determinar conclusiones definitivas en los desenlaces estudiados probablemente son mayores que los incorporados en varios de los ensayos analizados y además, es importante tener en cuenta que uno de los ensayos incluidos no solo incluyó cristaloides, sino también coloides, lo que podría haber sesgado su análisis[18].

En conclusión, los cristaloides balanceados se presentan como una alternativa con múltiples beneficios probables en la reanimación del paciente crítico por sobre los cristaloides no balanceados y por esta razón es que debería considerarse su uso por sobre los cristaloides no balanceados siempre y cuando no existan contraindicaciones para su utilización.

\section{Referencias}

1. Myburgh J, Mythen M. Resuscitation Fluids. New England Journal of Medicine. 2013;369(13):1243-1251. https://doi.org/10.1056/NEJMra1208627

2. Reddy $S$, Weinberg L, Young P. Crystalloid fluid therapy. Critical Care. 2016;20(1). https://doi. org/10.1186/s13054-016-12175

3. Yunos N, Kim I, Bellomo R, Bailey $M, H o L$, Story $D$ et al. The biochemical effects of restricting chloride-rich fluids in intensive care*. Critical Care Medicine. 2011;39(11):24192424. https://doi.org/10.1097/ CCM.0b013e31822571e5

4. Yunos N, Bellomo R, Hegarty
C, Story D, Ho L, Bailey M. Association Between a ChlorideLiberal vs Chloride-Restrictive Intravenous Fluid Administration Strategy and Kidney Injury in Critically III Adults. JAMA. 2012;308(15):1566. https://doi. org/10.1001/jama.2012.13356

5. Lobo D, Awad S. Should chloride-rich crystalloids remain the mainstay of fluid resuscitation to prevent 'pre-renal' acute kidney injury?: con. Kidney International. 2014;86(6):10961105. https://doi.org/10.1038/ ki.2014.105

6. Raghunathan K, Shaw A, Nathanson B, Stürmer T, Brookhart A, Stefan M et al. Association Between the Choice of IV Crystalloid and In-Hospital Mortality Among Critically III Adults
With Sepsis*. Critical Care Medicine. 2014;42(7):15851591. https://doi.org/10.1097/ CCM.0000000000000305

7. Hu J, Wang $Y$, Geng X, Chen R, Xu $X$, Zhang $X$ et al. Metabolic acidosis as a risk factor for the development of acute kidney injury and hospital mortality. Experimental and Therapeutic Medicine. 2017;13(5):23622374. https://doi.org/10.3892/ etm.2017.4292

8. Duffy R, Foroozesh M, Loflin R, le $S$, Icard B, Tegge A et al. Normal saline versus Normosol ${ }^{\mathrm{TM}} \mathrm{-R}$ in sepsis resuscitation: A retrospective cohort study. Journal of the Intensive Care Society. 2018;20(3):223-230. https://doi. rg/10.1177/1751143718786113

9. Chua H, Venkatesh B, Sta- 
chowski E, Schneider A, Perkins $\mathrm{K}$, Ladanyi $\mathrm{S}$ et al. Plasma-Lyte 148 vs $0.9 \%$ saline for fluid resuscitation in diabetic ketoacidosis. Journal of Critical Care. 2012;27(2):138-145. https://doi. org/10.1016/j.jcrc.2012.01.007

10. Jaynes M, Murphy $C$, Ali N, Krautwater A, Lehman A, Doepker B. Association between chloride content of intravenous fluids and acute kidney injury in critically ill medical patients with sepsis. Journal of Critical Care. 2018;44:363-367. https://doi. org/10.1016/j.jcrc.2017.12.014

11. Young J, Utter G, Schermer C, Galante J, Phan H, Yang $Y$ et al. Saline Versus PlasmaLyte $A$ in Initial Resuscitation of Trauma Patients. Annals of Surgery. 2014;259(2):255262. https://doi.org/10.1097/ SLA.0b013e318295feba

12. Verma B, Luethi $N$, Cioccari $L$, Lloyd-Donald P, Crisman M, Eastwood $G$ et al. A multicentre, randomised controlled pilot study of fluid resuscitation with saline or Plasma-Lyte 148 in critically ill patients. Critical Care and Resuscitation. 2016;18(3):205212.

13. Semler $M$, Self $W$, Wanderer J, Ehrenfeld J, Wang L, Byrne D et al. Balanced Crystalloids versus Saline in Critically III Adults. New England Journal of Medicine. 2018;378(9):829-839. https://doi.org/10.1056/NEJMoa1711584

14. Semler M, Wanderer J, Ehrenfeld J, Stollings J, Self W, Siew E et al. Balanced Crystalloids versus Saline in the Intensive Care Unit. The SALT Randomized Trial. American Journal of Respiratory and Critical Care Medicine. 2017;195(10):13621372. https://doi.org/10.1164/ rccm.201607-13450C

15. Young $P$, Bailey $M$, Beasley $R$, Henderson S, Mackle D, McAr- thur C et al. Effect of a Buffered Crystalloid Solution vs Saline on Acute Kidney Injury Among Patients in the Intensive Care Unit: The SPLIT Randomized Clinical Trial. JAMA. 2015;314(16):1701. https://doi.org/10.1001/ jama.2015.12334

16. Choosakul S, Harinwan K, Chirapongsathorn S, Opuchar K, Sanpajit T, Piyanirun W, et al. Comparison of normal saline versus Lactated Ringer's solution for fluid resuscitation in patients with mild acute pancreatitis, A randomized controlled trial. Pancreatology. 2018;18(5):507512. https://doi.org/10.1016/j. pan.2018.04.016

17. Wang $X$, Zhang $C$, Huang $G$, Han D, Meng X, Guo Y et al. Association between chloriderich versus chloride-restrictive intravenous fluid administration and acute kidney injury in cardiovascular patients in ICU wards. Experimental and Therapeutic Medicine. 2016;12(2):987990. https://doi.org/10.3892/ etm.2016.3431

18. de-Madaria E, Herrera-Marante I, González-Camacho V, Bonjoch L, Quesada-Vázquez N, AlmentaSaavedra I et al. Fluid resuscitation with lactated Ringer's solution vs normal saline in acute pancreatitis: A triple-blind, randomized, controlled trial. United European Gastroenterology Journal. 2017;6(1):63-72. https://doi. $\mathrm{rg} / 10.1177 / 2050640617707864$

19. Wu B, Hwang J, Gardner T, Repas K, Delee R, Yu S et al. Lactated Ringer's Solution Reduces Systemic Inflammation Compared With Saline in Patients With Acute Pancreatitis. Clinical Gastroenterology and Hepatology. 2011;9(8):710-717. e1. https://doi.org/10.1016/j. cgh.2011.04.026

20. Filis C, Vasileiadis I, Koutsoukou A. Hyperchloraemia in sepsis. Annals of Intensive Care. 2018;8(1). https://doi. org/10.1186/s13613-018-03884

21. Ronco C, Bellomo R, Kellum J. Acute kidney injury. The Lancet. 2019;394(10212):1949-1964. https://doi.org/10.1016/S01406736(19)32563-2

22. Petros A, Marshall J, van Saene $H$. Should morbidity replace mortality as an endpoint for clinical trials in intensive care?. The Lancet. 1995;345(8946):369371. https://doi.org/10.1016/ S0140-6736(95)90347-X

23. Veldhoen R, Howes D, Maslove D. Is Mortality a Useful Primary End Point for Critical Care Trials?. Chest. 2019. https://doi. org/10.1016/j.chest.2019.11.019

24. Ridgeon E, Bellomo R, Aberegg S, Sweeney R, Varughese R, Landoni $G$ et al. Effect sizes in ongoing randomized controIled critical care trials. Critical Care. 2017;21(1). https://doi. org/10.1186/s13054-017-1726-x

25. Van Regenmortel N, Verbrugghe W, Roelant E, Van den Wyngaert $T$, Jorens P. Maintenance fluid therapy and fluid creep impose more significant fluid, sodium, and chloride burdens than resuscitation fluids in critically ill patients: a retrospective study in a tertiary mixed ICU population. Intensive Care Medicine. 2018;44(4):409-417. https://doi. org/10.1007/s00134-018-5147-3

26. Hammond N, Bellomo R, Gallagher M, Gattas D, Glass P, Mackle $D$ et al. The Plasma-Lyte $148 \mathrm{v}$ Saline (PLUS) study protocol: a multicentre, randomised controlled trial of the effect of intensive care fluid therapy on mortality. Critical Care and Resuscitation. 2017;19(3):239-246.

27. Zampieri F, Azevedo L, Corrêa T, Falavigna M, Machado F, de Assunção T et al. Study protocol for the Balanced Solution versus 
Saline in Intensive Care Study (BaSICS): a factorial randomised trial. Crit Care Resusc. 2017;19(2):175-182.

28. Bolster L, Bosch M, Brownbridge B, Saxena A. RAP Trial: Ringer's Lactate and Packed Red Blood Cell Transfusion, An in Vitro Study and Chart Review. Blood. 2009;114(22):2105-2105. https://doi.org/10.1182/blood. V114.22.2105.2105

29. Lorenzo M, Davis J, Negin S, Kaups K, Parks S, Brubaker D et al. Can Ringer's Lactate Be Used
Safely with Blood Transfusions?. The American Journal of Surgery. 1998;175(4):308-310. https://doi.org/10.1016/500029610(98)00011-7

30. Albert K, van Vlymen J, James P, Parlow J. Ringer's lactate is compatible with the rapid infusion of AS-3 preserved packed red blood cells. Canadian Journal of Anesthesia/Journal canadien d'anesthésie. 2009;56(5):352356. https://doi.org/10.1007/ s12630-009-9070-5

31. Khajavi M, Etezadi F, Moharari R,
Imani F, Meysamie A, Khashayar $P$ et al. Effects of Normal Saline vs. Lactated Ringer's during Renal Transplantation. Renal Failure. 2008;30(5):535-539. https:// doi.1080/08860220802064770

32. O'Malley CMN, Frumento RJ, Hardy MA, et al. A randomized, double-blind comparison of lactated Ringer's solution and $0.9 \% \mathrm{NaCl}$ during renal transplantation. Anesth Analg. 2005;100:1518-1524. https://doi.org/10.1213/01. ANE.0000150939.28904.81 